\title{
Effects of phase duration on detection of electrical stimulation of the human cochlea
}

\author{
Andrew K. Moon, Teresa A. Zwolan and Bryan E. Pfingst \\ Kresge Hearing Research Institute, Department of Otolaryngology, University of Michigan Medical Center, Ann Arbor, Michigan, USA
}

(Received 24 September 1992; Revision received 18 January 1993; Accepted 20 January 1993)

\begin{abstract}
Detection thresholds for biphasic symmetric pulses were measured in fourteen human subjects implanted with the Cochlear Corporation Nucleus 22 Implant. The effects of phase duration on thresholds were studied using single pulses, and $500 \mathrm{~ms}$ pulse trains at 100 pps. Psychophysical detection thresholds decreased as a function of phase duration with a changc in slope at approximatcly 0.5 ms /phasc. Mcan single-pulse and pulse-train slopes were -3.60 and $-4.25 \mathrm{~dB} /$ doubling of phase duration for pulse durations of less than about $0.5 \mathrm{~ms} /$ phase. For pulse durations greater than $0.5 \mathrm{~ms} /$ phase, mean slopes were -5.71 and $-7.54 \mathrm{~dB}$ /doubling for single pulses and pulse trains, respectively. Thresholds for pulse trains decreased as a function of stimulus duration for durations up to at least $300 \mathrm{~ms}$, with the rate of decrease being dependent on the phase duration of the pulse. Effects of stimulus duration were greater for longer phase duration signals. We hypothesize that the longer phase duration pulses activate multiple spikes in a single fiber and/or more effective patterns of spikes across fibers, which may explain why slopes of psychophysical threshold functions are steeper than those of functions for single auditory nerve fibers for longer duration pulses. Thresholds were compared to respective speech perception scores (CID sentences) since thresholds for long phase duration signals have been shown previously to be correlated with nerve survival patterns, and nerve survival patterns may affect speech perception. Correlation coefficients ranged from -0.59 to -0.81 , depending on stimulus parameters and subject selection.
\end{abstract}

Auditory prosthesis; Electrical stimulation; Human; Psychophysics; Detection thresholds; Phase duration

\section{Introduction}

Electrical stimulation of the cochlea, via electrodes implanted in the scala tympani, produces sensations of sound, and the auditory information transmitted by such stimulations is sufficient to support prosthetic devices for the deaf. Over 3,000 profoundly deaf individuals currently use these cochlear prosthetic devices, but the quality of information received, as indicated, for example, by speech perception performance, varies widely from subject to subject (Gantz et al., 1988). Recently, significant improvements in speech perception scores have been achieved by alterations in processor design (Wilson et al., 1991). We assume that ideal design and calibration of these prosthetic devices would be facilitated by a better understanding of the neural mechanisms underlying detection and discrimination of the electrical stimuli. While those mechanisms are understood in general terms, many specifics, such as the exact pattern of neural activity required for detection of electrical stimulation, are not known. The study reported here is one of a series of investigations of the

Correspondence to: Bryan E. Pfingst, Kresge Hearing Research Institute, 1301 East Ann Street, Box 0506, University of Michigan Medical Center, Ann Arbor, MI 48109-0506 USA. Fax: (313) 764-0014. stimulus features important for detection of electrical stimulation of the deaf ear.

One question to be asked in these studies is to what extent the psychophysical detection of electrical stimuli can be accounted for by known properties of electrically stimulated nerve. Physiological studies have shown that phase duration of electrical signals is an important variable determining the current level sufficient to elicit neural action potentials (Hill, 1936; Frankenhaeuser and Huxley, 1964). Phase duration is the duration of each half cycle of the stimulus in which the current affecting the neurons is in one phase (positive or negative) and in which the neural membrane integrates charge over time until the threshold for discharge is reached. This study begins with determination of detection thresholds as a function of phase duration for single, biphasic, charge balanced pulses.

Single pulses are used for the initial experiment because they represent a relatively simple case for determining the effects of phase duration. Previous studies in humans and animals have shown that stimulus features other than phase duration, particularly pulse rate (Shannon, 1985; Pfingst and Morris, 1992) and stimulus duration (Shannon, 1989; Pfingst et al., 1991; Pfingst and Morris, 1992) have significant effects on detection thresholds, and that these variables can interact with phase duration. Thus, detection of sinu- 
soids and pulse trains is influenced by a combination of stimulus features. In subsequent experiments in this study, effects of these additional stimulus features are studied in conjunction with phase duration.

These studies were conducted using a population of human subjects with Nucleus 22 Cochlear Prostheses (Clark et al., 1987; Skinner, 1991). In these subjects, pulsatile stimuli are generated by implanted receiver/stimulator modules that drive electrodes placed in the scala tympani. For safety reasons, all stimuli are charge balanced biphasic pulses. The pulses have a unique characteristic waveform, which is described in the Methods section.

Previous studies in animals have shown that individual differences in thresholds across subjects are correlated with differences in nerve-survival patterns (Pfingst et al., 1985), although the mechanisms underlying these correlations are unknown. It has long been assumed that individual differences in more complex functions such as speech perception with cochlear implants are also due, in part, to differences in nerve-survival patterns across patients. These two considerations lead to the hypothesis that speech perception differences across subjects will be correlated with differences in detection thresholds. Previous studies using sinusoidal stimuli have shown that the best correlations between nerve-survival patterns and thresholds occur for thresholds measured using low frequency signals (around 100 $\mathrm{Hz}$ ). Subjects with the best nerve-survival patterns have lower thresholds at $100 \mathrm{~Hz}$, and threshold vs frequency functions with steeper slopes, than subjects with poorer nerve survival. Whether these differences in shape of the threshold functions are related to phase duration, rate, or a combination of stimulus features, has not been determined. Attempts to use thresholds to predict performance with cochlear implants have met with varying degrees of success (Kileny et al., 1991; Kuk et al., 1990; Blamey et al., 1992). In the experiments reported here we examined the relationship between single pulse and pulse train detection thresholds and speech perception test scores.

\section{Methods}

\section{Subjects and equipment}

Data were collected from fourteen postlingually profoundly deaf human subjects who had been implanted with the Cochlear Corporation Nucleus 22 Cochlear Implant System. Details about the subjects are given in Table I. The Nucleus prosthesis consisted of an electrode array that was surgically implanted in the scala tympani via the round window. There were 22 electrodes in the array with adjacent electrodes separated by $0.75 \mathrm{~mm}$ center to center. The electrodes were labelled 1-22, starting at the basal end of the array. The electrodes were driven by a receiver/stimulator implanted in the temporal bone. The receiver/stimulator was powered and programmed by a radio-frequency signal from a transmitter coil that was aligned and retained over the receiver/stimulator magnetically. Signals were sent to the transmitter coil from a Cochlear Corporation Dual Processor Interface (DPI) containing a Mini Speech Processor (MSP). The DPI was controlled by an IBM PC, fitted with Cochlear Corporation IF3 and IF4 boards, using the Nucleus Voice Input Promontory Stimulator (VIPS) software.

Temporal parameters of the stimuli were checked

TABLE I

Subjects listed in order of descending threshold level for $96 \mu \mathrm{sec} /$ phase single pulse stimuli (as shown in table II)

\begin{tabular}{|c|c|c|c|c|c|c|}
\hline $\begin{array}{l}\text { Subject } \\
\text { ID }\end{array}$ & Sex & Age & Tinnitus & $\begin{array}{l}\text { Duration of } \\
\text { profound } \\
\text { deafness prior } \\
\text { to implantation } \\
\text { (years) }\end{array}$ & $\begin{array}{l}\text { Duration of } \\
\text { prosthesis use } \\
\text { prior to these } \\
\text { experiments } \\
\text { (months) }\end{array}$ & $\begin{array}{l}\text { Flectrodes } \\
\text { used for } \\
\text { threshold } \\
\text { testing }\end{array}$ \\
\hline $\mathrm{MC}$ & $\mathrm{F}$ & 78 & $\mathrm{~N}$ & 4 & 10 & $14-16$ \\
\hline BT & $\mathrm{M}$ & 60) & $\mathrm{Y}$ & 8 & 32 & $15-17$ \\
\hline $\mathrm{GE}$ & $F$ & 49 & $Y$ & 42 & 55 & $12-14$ \\
\hline $\mathrm{MO}$ & $\mathrm{M}$ & 66 & $Y$ & 46 & 45 & $14-16$ \\
\hline MKe & $M$ & 67 & $\mathrm{~N}$ & 5 & 12 & $7-4$ \\
\hline CT & $\mathrm{M}$ & 61 & $\mathrm{Y}$ & 29 & 13 & $14-16$ \\
\hline $\mathrm{RH}$ & $M$ & 58 & $\mathrm{Y}$ & 3 & 17 & $12-14$ \\
\hline MD & $F$ & 68 & $Y$ & 1 & 17 & $12-14$ \\
\hline $\mathrm{BC}$ & $\mathrm{M}$ & 48 & $\mathrm{Y}$ & 2 & 50 & $10-12$ \\
\hline HP & $M$ & 50 & $\mathrm{Y}$ & 19 & 28 & $12-14$ \\
\hline MK1 & $\mathrm{F}$ & 40 & $\mathrm{Y}$ & 4 & 23 & $10-12$ \\
\hline $\mathrm{EW}$ & F & 67 & $\mathrm{Y}$ & 1 & 7 & $14-16$ \\
\hline $\mathrm{KF}$ & $\mathrm{F}$ & 43 & $\mathrm{~N}$ & 1 & 4 & $8-10$ \\
\hline PD & $\mathrm{M}$ & 32 & $\mathrm{~N}$ & 1 & 10 & $10-12$ \\
\hline
\end{tabular}


by monitoring the output of a VIPS receiver/stimulator, which was an external hardware device similar to the implanted receiver/stimulator. The VIPS receiver/stimulator was driven by the same hardware and software used to control the subjects' implanted receiver/stimulators. During calibration sessions, the temperature of the VIPS receiver/stimulator was held constant at $38^{\circ} \mathrm{C}$ by a heating pad. Voltage across a 1 $\mathrm{k} \Omega$ load at the output of the VIPS receiver/stimulator was monitored using a Tektronix 2230 digital storage oscilloscope.

\section{Psychophysical procedures}

Two different audiologic test procedures were used to obtain thresholds: a method of adjustment and a modified Hughson-Westlake (method of limits) procedure.

In the method of adjustment, subjects were instructed to set a current level control knob to a point at which they could definitely recognize a stimulus and then lower the level to the point at which the stimulus was barely audible. Subjects with chronic tinnitus were instructed to set the current to a level that made the stimulus barcly audible over the tinnitus. All subjects were familiar with setting detection levels by this method because it was the procedure routinely used for clinical calibration of processors. Although the method of adjustment was the quicker method to set detection level, its use was restricted by the VIPS software to $500 \mathrm{~ms}$ pulse trains. Presentation rate was once every $1 \mathrm{~s}$. The control knob was set to allow the current to be adjusted in increments of one current level unit (about $0.16 \mathrm{~dB}$ on average, as detailed below) with a small change in the knob.

The modified Hughson-Westlake method (Carhart and Jerger, 1959), a standard audiological procedure, was used to obtain thresholds for stimuli less than 500 $\mathrm{ms}$ in duration as well as some $500 \mathrm{~ms}$ pulse-train thresholds for comparison to thresholds obtained using the method of adjustment. In this procedure, the experimenter controlled the presentation of test signals. Subjects were asked to acknowledge detection of an audible signal by raising their hand or finger. Presentation rate was determined arbitrarily by the experimenter but was not more than once every $1 \mathrm{~s}$ or less than once every $5 \mathrm{~s}$. The experimenter increased the level of current in five current-level steps until the subject could hear three consecutive signals then decreased the current to an inaudible level. Current was then increased by single unit steps until the subject could hear three consecutive signals. The threshold was calculated by subtracting three current level units from the final setting.

For both procedures, the stimulus was begun at the lowest current level setting, which ranged between 20 and $24 \mu \mathrm{A}$ peak depending on the particular implanted receiver/stimulator being used. Stimuli of different phase durations or stimulus durations were presented in random order, and all stimuli in the series werc run before starting a new random series. Five series were run so that five detection levels for each different stimulus were recorded. The mean threshold was calculated for each of the stimuli.

\section{Stimulus description studies}

Subjects were asked to describe the stimuli to which they were responding. For this purpose, single pulse and 100 pps $500 \mathrm{~ms}$ pulse train stimuli were presented at short and long phase durations: 96 and 768 or 1536 $\mu \mathrm{s} /$ phase. Interstimulus interval was $500 \mathrm{~ms}$. All signals were presented at the previously determined threshold levels. Subjects were first asked to describe

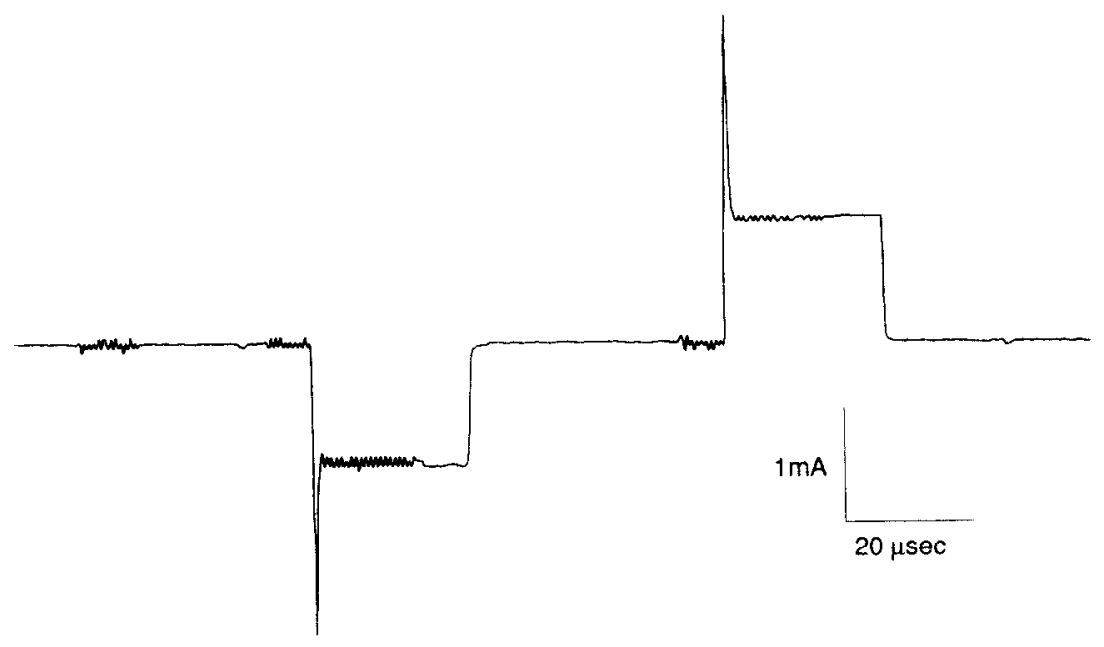

Fig. 1. Plot of a typical pulse produced by the Nucleus VIPS receiver/stimulator. Phase duration was set at $24 \mu \mathrm{s} / \mathrm{phase}$ and amplitude was adjusted to about $1 \mathrm{~mA}$ peak. Voltage was measured across a $1 \mathrm{k} \Omega$ resistor at the output of the VIPS stimulator. Small-amplitude radio-frequency noise can be seen preceding and during each phase of the pulse. Due to the relatively low sampling rate of the oscilloscope, all frequencies generated in this high frequency noise are not represented in the plot. 
their perceptions and then were asked if the stimuli were heard or felt.

\section{Speech tests}

CID Everyday Sentences were used to evaluate the subjects' auditory-alone speech recognition. Test stimuli were presented to subjects via a loud speaker at their most comfortable listening level (MCL) while they were seated in a sound-attenuated chamber. Subjects wore their processors in a normal fashion. MCL was set by adjusting the level of a sample sentence. The test consisted of 20 taped sentences containing 100 key words administered in an open-set manner. Subjects were asked to write whatever words they could recognize following presentation of each sentence. A percentage-correct score was derived from the key words in the test.

\section{Stimuli and experimental design}

Symmetric biphasic rectangular pulses were used in all experiments. The initial phase to the more basal electrode was always negative. Pulse durations ranged from 12 to $4344 \mu \mathrm{s} /$ phase. However, since the limits of the current deliverable by the implanted receiver/ stimulators ranged from a minimum of $20-24 \mu \mathrm{A}$ peak to a maximum of $1.5-1.8 \mathrm{~mA}$ peak, not all subjects could be tested at all pulse widths.

A typical pulse is illustrated in Fig. 1. There were three special features characteristic of all pulses. First, there was an overshoot at the beginning of each phase in the pulse that was independent of the pulse duration or amplitude. With a $1 \mathrm{k} \Omega$ load on the VIPS receiver/stimulator the amplitude of this overshoot was measured at $2.7 \mathrm{~mA}$, and had a duration of less than 1 $\mu \mathrm{s}$. Second, high frequency noise bursts from the transmitter occurred before and during each pulse. Third, there was a $40 \mu \mathrm{s}$ gap between negative and positive phases of the pulse.

Current level was controlled by the VIPS software. There were 238 usable current levels. The current at each level was determined by Cochlear Corporation prior to implantation of each receiver/stimulator. Calibration tables from Cochlear Corporation were used to translate the stimulus level units to microamperes. The step size in $\mathrm{dB}$ of current was variable from step to step but the average step size was about $0.16 \mathrm{~dB}$, and at least $95 \%$ of step sizes were less than $0.45 \mathrm{~dB}$.

The electrode pairs used for threshold testing are given in Table I. These pairs were determined by selecting an electrode pair that displayed a typical dynamic range in the array and that was not closer than 5 electrodes basally or apically to the ends of the array. All pulse thresholds were measured at a bipolar $+1(\mathrm{BP}+1)$ configuration. In the $\mathrm{BP}+1$ mode, one inactive electrode separated the two stimulated electrodes, so the separation between the two stimulated electrodes was $1.5 \mathrm{~mm}$ center to center. Speech perception tests were conducted at the subjects' normal electrode configuration, which was $\mathrm{BP}+1$ for all subjects except subject $\mathrm{RH}$ whose normal spacing was bipolar $+2(\mathrm{BP}+2)$. In $\mathrm{BP}+2$, the stimulated electrodes were separated by $2.25 \mathrm{~mm}$ center to center. Subjects utilized the Multi-Peak encoding strategy (Skinner et al., 1991) during the speech perception tests.

Experiment 1 used single pulses, i.e. subjects were asked to detect a single, biphasic pulse on each trial. The modified Hughson-Westlake procedure was used for all of the subjects.

In Experiment 2, subjects were asked to detect pulse trains. Pulse train parameters used were $500 \mathrm{~ms}$ stimulus duration at 100 pps. The method of adjustment was the primary psychophysical procedure used. During Experiments 1 and 2, subjects were asked to describe their perceptions of the single pulses and pulse trains.

Experiment 3 focused on effects of stimulus duration and used pulse trains with a discrete number of pulses ranging from 1 to 30 pulses. Two phase durations were used: 96 and $1536 \mu \mathrm{s} /$ phase.

In Experiment 4, thresholds for $500 \mathrm{~ms}$ pulse trains at 100 pps were measured using the method of adjustment and the modified Hughson-Westlake procedure, alternately in the same session.

Speech perception was tested as part of the subject's clinical evaluation. Subjects were tested approximately twice every year. The most recent scores available were used for comparisons to thresholds in this study.

\section{Results}

\section{Effects of phase duration}

\section{Single pulses}

Threshold vs phase duration functions for single pulse signals for the fourteen subjects are shown in Fig. 2 . The longest pulse duration at which we could test all subjects was $768 \mu \mathrm{s}$ /phase. For that stimulus, thresholds covered a $21 \mathrm{~dB}$ range across subjects from -27.13 to $-6.05 \mathrm{~dB}$ re $1 \mathrm{~mA}$ peak (44 to $498 \mu \mathrm{A}$ peak) (See Table II). The majority of subjects' thresholds (excluding the two subjects with the highest and lowest thresholds) fell within an $8 \mathrm{~dB}$ range at this pulse duration. The shortest pulse duration at which we could test all subjects was $96 \mu \mathrm{s} /$ phase. Thresholds for $96 \mu \mathrm{s} /$ phase pulses covered a $13 \mathrm{~dB}$ range from $-11.68 \mathrm{~dB}$ and $+1.37 \mathrm{~dB}$ re $1 \mathrm{~mA}$ peak (about 261 to $1171 \mu \mathrm{A}$ peak).

Reliability of threshold measurements was determined by examining individual subjects' standard deviations over five threshold measurements for each phase duration. The mean standard deviation across all subjects for all single pulse stimuli was $0.50 \mathrm{~dB}$. In 71 cases 


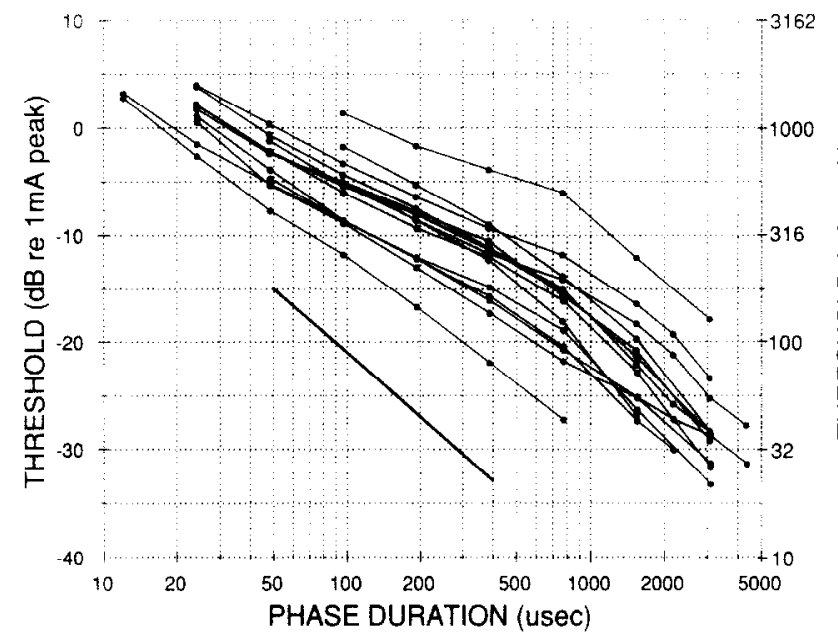

Fig. 2. Psychophysical detection thresholds plotted as a function of phase duration for single pulse stimuli for 14 subjects. Each data point gives the mean of five rcpeated threshold measurements. See Tables I and II for individual subject identification. The bold line (lower left) illustrates a slope of $-6 \mathrm{~dB}$ /doubling of phase duration, indicating constant charge.

(73\%), standard deviation was less than $0.50 \mathrm{~dB}$. One subject MKe had particularly high standard deviations, averaging $1.88 \mathrm{~dB}$. Excluding data for this individual, the mean standard deviation for the remaining subjects was $0.37 \mathrm{~dB}$.

The threshold vs phase duration functions had negative slopes with slopes becoming steeper for phase durations longer than $0.5 \mathrm{~ms}$ (See Table III). At pulse durations of less than $0.5 \mathrm{~ms} /$ phase, the mcan slope was $-3.60 \mathrm{~dB} /$ doubling of phase duration while for longer pulse durations, mean slope was -5.71 $\mathrm{dB} /$ doubling. A paired-samples $t$-test run on columns
TABLE II

Single pulse and pulse train detection thresholds in $\mathrm{dB}$ re $1 \mathrm{~mA}$ peak for two of the pulse durations tested. Subjects are listed in order of decreasing threshold for single pulses at $96 \mu \mathrm{s}$ /phase

\begin{tabular}{|c|c|c|c|c|}
\hline \multirow[t]{2}{*}{ Subject } & \multicolumn{2}{|c|}{$96 \mu \mathrm{s} /$ phase } & \multicolumn{2}{|c|}{$768 \mu \mathrm{s} /$ phase } \\
\hline & $\begin{array}{l}\text { single } \\
\text { pulses }\end{array}$ & $\begin{array}{l}\text { pulse } \\
\text { trains }\end{array}$ & $\begin{array}{l}\text { single } \\
\text { pulses }\end{array}$ & $\begin{array}{l}\text { pulse } \\
\text { trains }\end{array}$ \\
\hline $\mathrm{MC}$ & +1.37 & -1.77 & -6.05 & -10.97 \\
\hline BT & -1.75 & -2.75 & -13.81 & -18.71 \\
\hline $\mathrm{GE}$ & -3.30 & -4.25 & -11.84 & -15.64 \\
\hline MO & -4.35 & -6.32 & -14.98 & -21.05 \\
\hline MKe & -5.00 & -4.97 & -15.63 & -16.37 \\
\hline $\mathrm{CT}$ & -5.03 & -6.88 & -18.06 & -23.45 \\
\hline RH & -5.32 & -4.79 & -14.13 & -14.11 \\
\hline MD & -5.34 & -5.98 & -15.26 & -19.95 \\
\hline $\mathrm{BC}$ & -5.94 & -6.64 & -16.02 & -22.36 \\
\hline $\mathrm{HP}$ & -8.47 & -9.25 & -20.33 & -20.61 \\
\hline MK1 & -8.54 & -9.22 & -20.63 & -21.03 \\
\hline $\mathrm{EW}$ & -8.54 & -9.92 & -18.79 & -23.02 \\
\hline $\mathrm{KF}$ & -8.78 & -9.84 & -21.71 & -23.93 \\
\hline PD & -11.68 & -14.57 & -27.13 & -29.37 \\
\hline
\end{tabular}

A and B in Table III allowed us to reject the null hypothesis of no difference in slopes of the functions for the long vs short duration pulses $(t=4.43, d f=12$, $\mathrm{p}=0.001$ ).

The shapes of the threshold vs phase duration functions varied slightly from subject to subject. Of 13 cases where we could measure slopes for pulses greater than $768 \mu \mathrm{s} /$ phase, $9(69 \%)$ showed slope (absolute value) increases of greater than $1 \mathrm{~dB} /$ doubling as phase duration increased above $0.5 \mathrm{~ms} /$ phase (see Table III, column 6 ). The remaining 4 cases $(31 \%)$ showed only minor increases or decreases in slope. The point on the

\section{TABLE III}

Slopes in $\mathrm{dB}$ /doubling of phase duration of threshold functions shown in Figs. 2 and 4. Subjects are listed in same order as in Table II

\begin{tabular}{|c|c|c|c|c|c|c|c|c|}
\hline \multirow[b]{2}{*}{ ( $\mu \mathrm{s} /$ phase) } & \multicolumn{2}{|c|}{ Single pulses } & \multicolumn{2}{|c|}{ Pulse trains } & \multirow[t]{2}{*}{ A-B } & \multirow[t]{2}{*}{$C-D$} & \multirow[t]{2}{*}{$A-C$} & \multirow[t]{2}{*}{$\mathrm{B}-\mathrm{D}$} \\
\hline & $\begin{array}{l}\text { A } \\
12-384\end{array}$ & $\begin{array}{l}\text { B } \\
768.4344\end{array}$ & $\begin{array}{l}C \\
12-384\end{array}$ & $\begin{array}{l}\mathrm{D} \\
768-4344\end{array}$ & & & & \\
\hline \multicolumn{9}{|l|}{ Subject } \\
\hline $\mathrm{MC}$ & -2.63 & -5.92 & -5.92 & -5.89 & 3.29 & -0.03 & 3.29 & -0.03 \\
\hline BT & -3.62 & -4.77 & -7.26 & -7.49 & 1.15 & 0.23 & 3.64 & 2.72 \\
\hline GE & -3.27 & -5.64 & -3.33 & -7.36 & 2.37 & 4.03 & 0.06 & 1.72 \\
\hline $\mathrm{MO}$ & -3.67 & -6.81 & -4.10 & -6.95 & 3.14 & 2.85 & 0.43 & 0.14 \\
\hline $\mathrm{MKe}$ & -3.00 & -6.81 & -2.41 & -7.70 & 3.81 & 5.29 & -0.59 & 0.89 \\
\hline $\mathrm{CT}$ & -3.65 & -8.81 & -4.34 & & 5.16 & & 0.69 & \\
\hline RH & -3.49 & -3.16 & -3.28 & -7.73 & $\cdots 0.33$ & 4.45 & 0.21 & 4.57 \\
\hline $\mathrm{MD}$ & -3.28 & -6.11 & -3.78 & -8.98 & 2.83 & 5.20 & 0.50 & 2.87 \\
\hline $\mathrm{BC}$ & -3.29 & -7.69 & -3.48 & -9.78 & 4.40 & 6.30 & 0.19 & 2.09 \\
\hline HP & -4.19 & -6.48 & -4.63 & & 2.29 & & 0.44 & \\
\hline MK1 & -3.77 & -4.16 & -3.72 & -5.05 & 0.39 & 1.33 & -0.05 & 0.89 \\
\hline $\mathrm{EW}$ & -3.38 & -3.61 & -3.69 & -9.12 & 0.23 & 5.43 & 0.31 & 5.51 \\
\hline KF & -4.33 & -4.25 & -4.72 & -6.85 & -0.08 & 2.13 & 0.39 & 2.60 \\
\hline PD & -4.85 & & -4.86 & & & & 0.01 & \\
\hline Mean & -3.60 & -5.71 & -4.25 & -7.54 & 2.20 & 3.38 & 0.65 & 2.18 \\
\hline
\end{tabular}


threshold vs phase duration function where slope increased varied slightly from subject to subject. In 7 cases, there was an increase in slope starting at 384 $\mu \mathrm{s} /$ phase. Four cases showed the first increase in slope at $768 \mu \mathrm{s} /$ phase. Several cases showed an initial increase in slope at $384 \mu \mathrm{s} /$ phase and additional increases at higher phase durations.

The data from Fig. 2 are replotted in Fig. 3 showing total charge per phase at threshold as a function of phase duration. Maximum charge ranged from about 33 to $392 \mathrm{nC} /$ phase. Charge per phase at threshold increased as a function of phase duration up to a peak at between 768 and $4344 \mu \mathrm{s} /$ phase and then, in most cases, decreased with further increases in phase duration. The inflection points in these curves, where slopes change from positive to negative, indicate the phase durations at which the slope of the threshold current vs phase duration functions change from a slope of less than $6 \mathrm{~dB}$ per doubling of phase duration to more than $6 \mathrm{~dB} /$ doubling. When the slope is less than $6 \mathrm{~dB} / \mathrm{dou}-$ bling, the system is acting as a leaky integrator, imperfectly integrating current over the length of the pulse, so more charge is required for longer pulses. When the slope of the threshold vs phase duration function exceeds $6 \mathrm{~dB}$ of current per doubling of phase duration, less charge is needed to reach threshold for longer pulses than for shorter pulses.

\section{Pulse trains}

Fig. 4 shows threshold vs phase duration functions for $500 \mathrm{~ms}$ pulse trains at $100 \mathrm{pps}$. For most subjects, thresholds for pulse trains were lower than those for single pulses. The difference between the threshold levels for single pulses and pulse trains generally increased as a function of phase duration. Data for two phase durations are shown in Table II. For $96 \mu \mathrm{s} /$ phase pulses, thresholds for pulse trains were an average of $1.18 \mathrm{~dB}$ lower than those for single pulses. A paired-

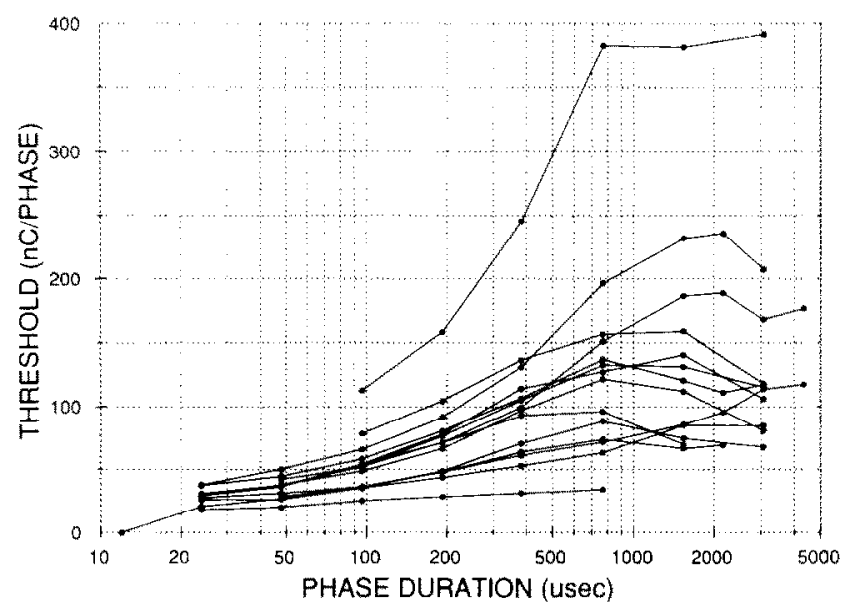

Fig. 3. Threshold data trom Fig. 2 replotted in units of charge per phase.

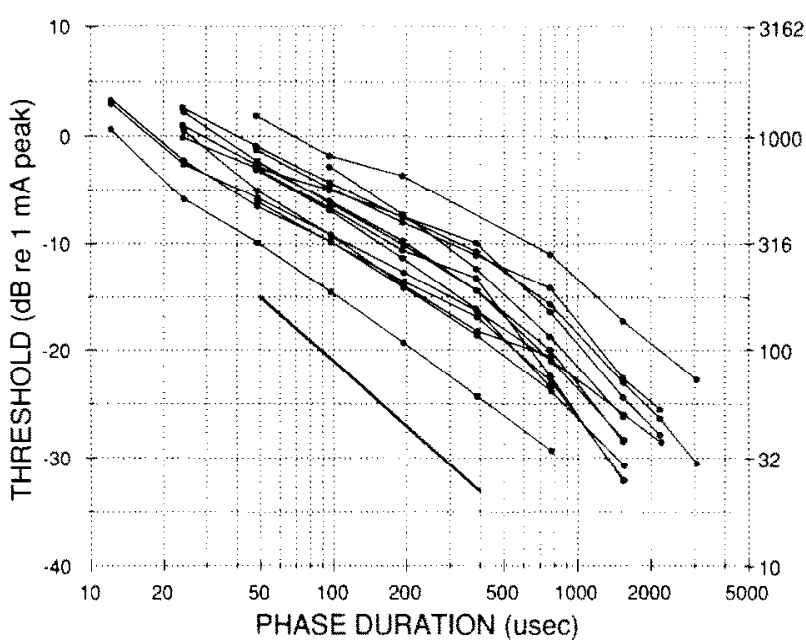

Fig. 4. Psychophysical detection thresholds plotted as a function of phase duration for pulse train stimuli. Subjects were the same as in Fig. 2. Stimuli were $500 \mathrm{~ms}$ in duration at $100 \mathrm{pps}$. The bold line (lower left) illustrates a slope of $-6 \mathrm{~dB} /$ doubling of phase duration.

samples $t$-test indicated that this difference was significantly different from zero $(t=4.36, d f=13, p=0.001)$. For $768 \mu \mathrm{s} /$ phase pulses, the difference in threshold between single pulses and pulse trains averaged 3.30 $\mathrm{dB}(\mathrm{t}=5.44, \mathrm{df}=13, P<0.001)$.

Standard deviations of repeated threshold measurements for pulse trains were slightly larger than those for single pulses. The mean standard deviation across all subjects for all stimuli in Experiment 2 was $0.62 \mathrm{~dB}$. One subject, MKe, had mean standard deviations greater than $1 \mathrm{~dB}$. With data for MKe removed, mean standard deviation across subjects was $0.46 \mathrm{~dB}$.

Threshold vs phase duration functions for pulse trains exhibited slightly greater negative slopes than those for single pulses (Table III). Slopes of threshold vs phase duration functions were compared for pulses less than $0.5 \mathrm{~ms} /$ phase (Table III columns A and C, and for pulses greater than $0.5 \mathrm{~ms} /$ phase (Table III, columns B and D) using paired-samples $t$-tests. For the shorter phase duration pulses the differences in slopes between the functions for single pulse and those for pulse trains was small, averaging $0.65 \mathrm{~dB} /$ doubling and we were not able to reject the null hypothesis that this difference was significantly different from zero $(t=$ $1.96, \mathrm{df}=13, \mathrm{p}=0.071)$. For the longer pulses the differences were larger, averaging $2.28 \mathrm{~dB} /$ doubling and were significantly different from zero $(t=4.15$, $\mathrm{df}=10, \mathrm{p}=0.002$ ).

Similar to the single pulse threshold functions, threshold vs phase duration functions for pulse trains exhibited a change in slope at around $0.5 \mathrm{~ms} /$ phase in most cases. For phase durations shorter than $0.5 \mathrm{~ms}$, the mean function slope was $-4.25 \mathrm{~dB} /$ doubling. The mean slope for longer durations was $-7.54 \mathrm{~dB} / \mathrm{dou}$ bling. Comparison of the slopes for pulses less than 0.5 $\mathrm{ms}$ /phase with those for pulses greater than 0.5 
$\mathrm{ms} /$ phase(Table III, columns $\mathrm{C}$ and D) using a paired-samples $t$-test showed that the slope differences were significantly different from zero $(t=5.08, \mathrm{df}=10$, $P<0.001$ ).

Like single pulse threshold vs phase duration functions, functions for pulse trains varied slightly in shape from subject to subject. Of the 11 cases where we could measure slopes for pulses greater than $768 \mu \mathrm{s} /$ phase, $9(82 \%)$ showed slope (absolute value) increases of greater than $1 \mathrm{~dB} /$ doubling as phase duration increased above $0.5 \mathrm{~ms} /$ phase. In 12 cases, the first increase in slope occurred at $384 \mu \mathrm{s} /$ phase. For one subject, the first increase occurred at $768 \mu \mathrm{s} /$ phase, and for another subject, the slope change could not be determined.

\section{Effects of Stimulus Duration}

Fig. 5 shows thresholds collected for five subjects in Experiment 3. Thresholds decreased as a function of stimulus duration. Single pulse thresholds, which are not shown in this figure, were an average of $0.65 \mathrm{~dB}$ higher than two-pulse thresholds. Slopes of threshold vs stimulus duration functions were steeper for longer phase duration stimuli. Mean slope for $1536 \mu \mathrm{s} /$ phase stimuli was $-0.81 \mathrm{~dB} /$ doubling of stimulus duration, while mean slope for $96 \mu \mathrm{s} /$ phase stimuli was -0.22 $\mathrm{dB} /$ stimulus doubling. Single pulse thresholds were not used in determining these slopes. A paired-samples $t$-test comparing the slopes of the threshold vs stimulus duration functions for short ( $96 \mu \mathrm{s} /$ phase) vs long (1536 $\mu \mathrm{s} /$ phase) pulses allowed us to reject the null hypothesis of no difference $(t=3.94, \mathrm{df}=4, \mathrm{p}=0.017)$.

\section{Effects of Psychophysical Procedure}

The two psychophysical procedures used in this study, the method of adjustment and the modified Hughson-Westlake procedure were compared using pulse train thresholds in three subjects. All pulse trains were produced at $100 \mathrm{pps}$ and $500 \mathrm{~ms}$ duration. Threshold functions are shown in Fig. 6. Functions obtained with the two procedures were similar.

\section{Descriptions of Stimulus Perception}

Subjects reported that the stimuli they were responding to were auditory in nature. When asked further if the stimuli were heard or felt, all subjects reported that the stimuli were heard. For single pulses near threshold, subjects reported that the signals sounded like a 'click', and they found it difficult to distinguish pulses of different durations. For pulse trains, subjects described long phase duration pulses as different from short phase duration pulses, though the descriptions of each varied widely from subject to
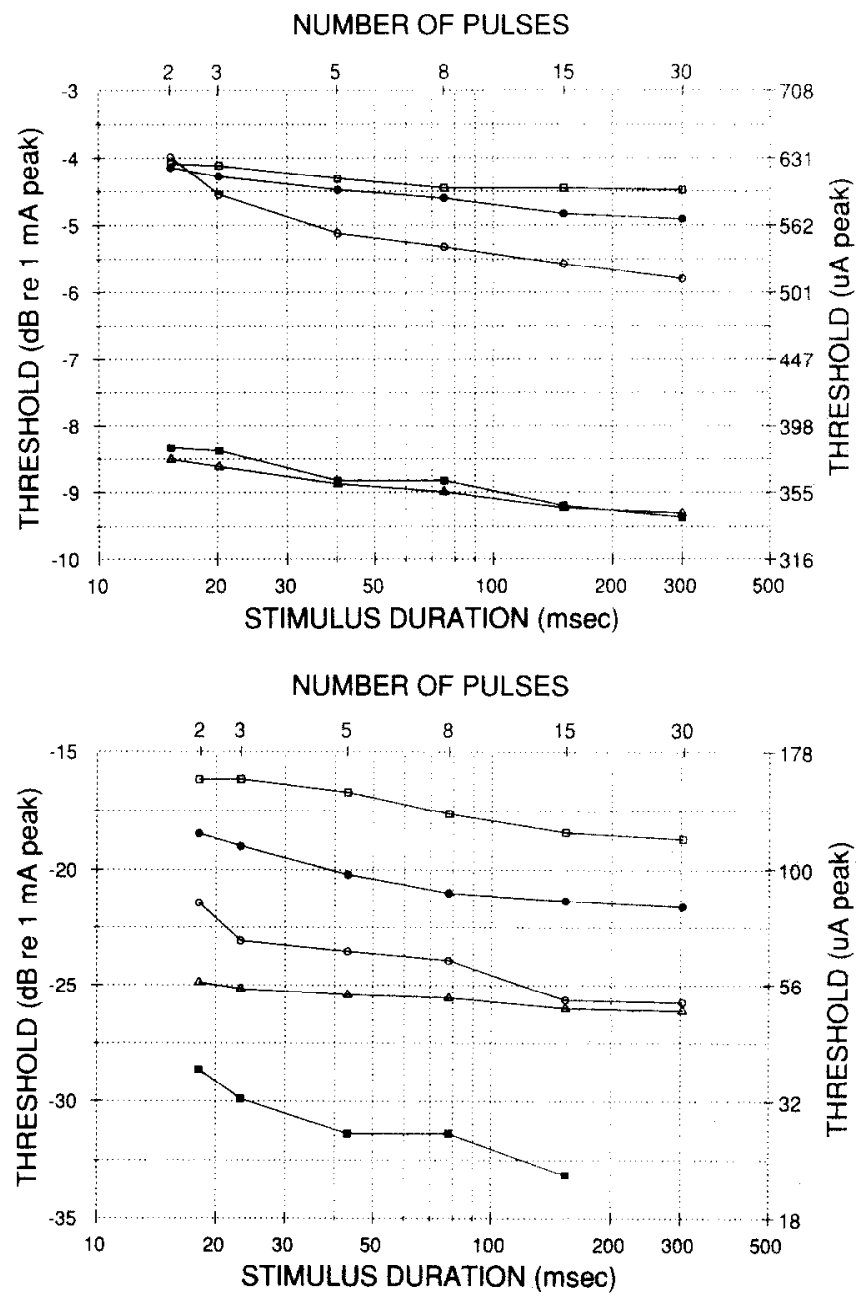

Fig. 5. Psychophysical detection thresholds plotted as a function of stimulus duration. Number of pulses in each stimulus is indicated on the top axis. Threshold functions for $96 \mu \mathrm{s} /$ phase stimuli are shown in the upper graph. Functions for $1536 \mu \mathrm{s} /$ phase stimuli are shown in the lower graph.

subject. Some typical descriptions for pulse trains were: 'buzzing', 'sawing', and 'large bell sound.' Perceived pitch at different phase durations varied from subject to subject.

\section{Speech Perception}

In Fig. 7, speech perception scores from the CID sentence test are compared to absolute thresholds for $1536 \mu \mathrm{s} /$ phase pulse trains obtained from the same subjects. Thresholds were available for only 11 subjects at this pulse duration. The correlation for all 11 subjects was -0.63 , which was statistically significant $(P<$ 0.05 ). If the two subjects (GE and MO) with the longest durations of deafness (greater than 30 years) were excluded from the analysis, the correlation was -0.81 . Correlations of CID sentence scores with 


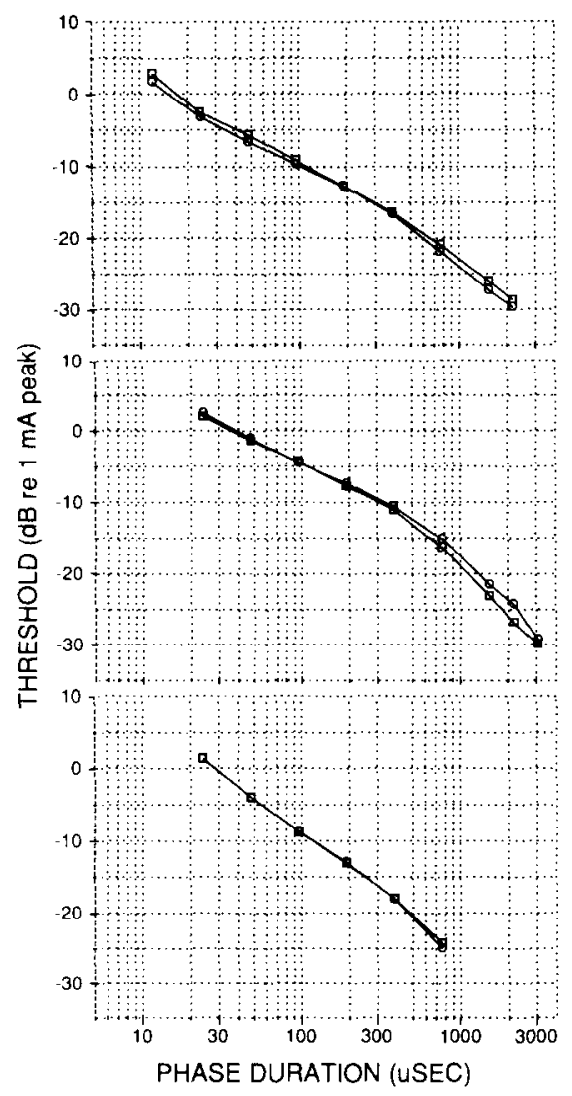

Fig. 6. Detection thresholds obtained by two psychophysical procedures: a method of adjustment (square symbols) and a modified Hughson-Westlake method (circles). Subjects were, from top, MKl, $\mathrm{GE}$, and $\mathrm{HP}$

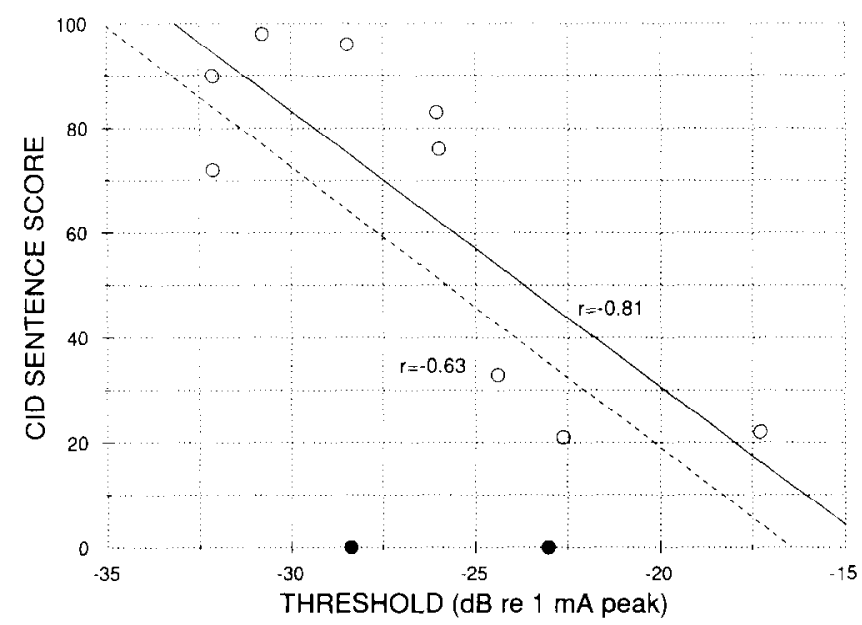

Fig. 7. Speech perception test scores compared to threshold levels for $1536 \mu \mathrm{s} /$ phase pulse trains for 11 subjects. See Table II for subject identification. CID Everyday Sentences were used in the speech perception tests. Filled circles represent subjects (GE and MO) with durations of profound deafness greater than 30 years. Best fit (least-squared deviation) straight lines are shown. The solid line and accompanying corrclation coefficient represent data in which $\mathrm{GE}$ and MO were omitted. The dashed line represents all subjects for which thresholds were available for this phase duration.
TABLE IV

Correlation coefficients for CID test scores vs threshold levels

\begin{tabular}{cll}
\hline & $\begin{array}{l}\text { All } \\
\text { available } \\
\text { subjects }\end{array}$ & $\begin{array}{l}\text { All subjects } \\
\text { with }<30 \text { years } \\
\text { profound deafness }\end{array}$ \\
\hline $\begin{array}{l}\text { Single pulse } \\
96 \mu \mathrm{sec} / \text { phase }\end{array}$ & -0.59 & -0.59 \\
$768 \mu \mathrm{sec} /$ phase & -0.62 & -0.62 \\
$1536 \mu \mathrm{sec} /$ phase & -0.62 & -0.66 \\
Pulse train & & \\
$96 \mu \mathrm{sec} /$ phase & -0.62 & -0.70 \\
$768 \mu \mathrm{sec} /$ phase & -0.63 & -0.78 \\
$1536 \mu \mathrm{sec} /$ phase & -0.63 & -0.81 \\
\hline
\end{tabular}

thresholds obtained for other stimuli are given in Table IV.

\section{Discussion}

\section{Summary of Results}

The major findings from this study are:

1. Thresholds decrease as a function of pulse duration. The rate of decrease depends on pulse duration, being greater at durations greater than $0.5 \mathrm{~ms} /$ phase (up to at least $4.3 \mathrm{~ms} /$ phase) than at durations less than 0.5 $\mathrm{ms} /$ phase.

2. Thresholds decrease as a function of stimulus duration. Thresholds for pulse trains are lower than those for single pulses.

3. Phase duration and stimulus duration interact in determining thresholds. The rate of threshold decrease as a function of pulse duration is greater for pulse trains than for single pulses. The rate of threshold decrease as a function of stimulus duration is grcater for long duration pulses than for short duration pulses. 4. Slopes of psychophysical threshold vs phase duration functions for pulses shorter than $0.5 \mathrm{~ms} /$ phase are similar to those reported for single auditory nerve fibers: about $-3.5 \mathrm{~dB} /$ doubling of phase duration. For pulses greater than $0.5 \mathrm{~ms} /$ phase, slopes of the psychophysical threshold functions $(-5.7$ to $-7.5 \mathrm{~dB}$ per doubling) are greater than those seen for single auditory nerve tibers. These relationships to published neurophysiological data are discussed below.

5. Absolute threshold levels for pulses less than 0.5 $\mathrm{ms} /$ phase are similar to those reported for single auditory nerve fibers, but levels of psychophysical thresholds for pulses greater than $0.5 \mathrm{~ms} /$ phase are lower than those for single auditory nerve fibers. These relationships are also discussed below.

6. Psychophysical detection threshold levels for pulses are correlated with intersubject differences in speech perception with cochlear prostheses. 


\section{Qualifications}

Several factors must be considered in estimating the generality of the results obtained in this study. One set of considerations concerns unique features of the pulses generated by the Nucleus 22 receiver/stimulator. We estimate that the radio-frequency signal has little effect on detection thresholds because it is a very high frequency (very short phase duration) signal and thresholds for such a signal would be very high. Similarly, the overshoot seen at the beginning of cach pulse is short in phase duration and thus should have a very high threshold. Neither of these aspects of the signal were detected by the subjects once the amplitude of the pulse was decreased below the subject's detection threshold. This fact does not rule out the possibility that these aspects of the signal might influence the detection thresholds of the pulses, but we estimate that these effects are minimal. Observations we made in a nonhuman primate subject with a hard-wired scala tympani implant indicated no significant difference in thresholds for pulses with an overshoot compared to those with no overshoot. In that experiment, the overshoot was created by peaking the input amplifier of a constant current stimulator to approximate the overshoot produced by the Nucleus VIPS receiver/stimulator. In the no-overshoot (normal) condition, peaking was disabled. Thresholds were measured with and without the overshoot for two pulse widths, 48 $\mu \mathrm{s} /$ phase and $1536 \mu \mathrm{s} /$ phase. Mean thresholds for the two conditions (with and without overshoot) differed by no more than $0.2 \mathrm{~dB}$ and these differences were not statistically significant.

Studies were done in one nonhuman primate subject to estimate the effect of the $40 \mu \mathrm{s}$ gap between the negative and positive phases of the pulse. We found the greatest effect of this gap at the shortest pulse duration tested ( $12 \mu \mathrm{s} /$ phase), where thresholds with the $40 \mu$ s gap present were $3 \mathrm{~dB}$ lower than when the gap was zero (for both single pulses and pulse trains). The effect of the gap decreased as a function of phase duration of the pulses, being almost zero for 96 $\mu \mathrm{s} /$ phase pulses. Thus the effect of the gap was a decrease in slope of the threshold vs phase duration function by about $1 \mathrm{~dB}$ /doubling for pulses less than $96 \mu \mathrm{s} /$ phase. It is unlikely that the interphase gap in the pulses was solely responsible for lower slopes of the threshold vs phase duration functions for pulses less than $0.5 \mathrm{~ms} /$ phase since such slopes have been seen previously for single pulses in nonhuman primates (Pfingst et al., 1991) and for pulse trains in a human subject (Shannon, 1983) using stimuli with no gap between the pulse phases.

Another factor to be considered is reliability of the data. This was assessed within sessions by repeated measures within subjects. We found that variability within subjects was low. Standard deviations over 5 trials averaged $0.5 \mathrm{~dB}$ for single pulses and $0.62 \mathrm{~dB}$ for

\section{TABLE V}

Mean slope of threshold vs phase duration functions (in $\mathrm{dB}$ /doubling of phase duration) for single pulses

\begin{tabular}{|c|c|c|c|c|c|c|}
\hline & \multicolumn{3}{|c|}{$<0.5 \mathrm{~ms} /$ phase } & \multicolumn{3}{|c|}{$>0.5 \mathrm{~ms} /$ phase } \\
\hline & Mean & $s$ & $N$ & Mean & $s$ & $N$ \\
\hline \multicolumn{7}{|l|}{$\begin{array}{l}\text { Psychophysical thresholds } \\
\text { humans }\end{array}$} \\
\hline $\begin{array}{l}\text { this study } \\
\text { macaque monkeys }\end{array}$ & -3.60 & 0.565 & 14 & -5.71 & 1.660 & 13 \\
\hline Pfingst et al., 1991 & -4.10 & 0.866 & 3 & -6.23 & 1.464 & 12 \\
\hline bipolar cases & - & & -- & -5.30 & 0.265 & 3 \\
\hline
\end{tabular}

\begin{tabular}{|c|c|c|c|c|c|c|}
\hline Mean slope of threshold vs phase & function & doublin & dur & 00 PPS, & trains & \\
\hline & $<0.5 \mathrm{~m}$ & & & $>0.5 \mathrm{n}$ & & \\
\hline & Mean & $s$ & $N$ & Mean & $s$ & $N$ \\
\hline $\begin{array}{l}\text { Psychophysical thresholds } \\
\text { humans }\end{array}$ & & & & & & \\
\hline this study & -4.25 & 1.215 & 14 & -7.54 & 1.394 & 11 \\
\hline Shannon et al., 1990 & -4.65 & 0.498 & 7 & -7.09 & 2.638 & 7 \\
\hline Shannon, 1983 & -4.5 & - & 1 & -8.5 & - & 1 \\
\hline Neural threshold & & & & & & \\
\hline squirrel monkeys & & & & & & \\
\hline Parkins and Colombo, 1987 & -4.33 & - & * & -1.69 & - & * \\
\hline cats & & & & & & \\
\hline Kiang and Moxon, 1972 & -4.61 & - & $* *$ & -2.61 & - & ** \\
\hline Javel et al., 1987 & -5.99 & 0.516 & 2 & - & - & - \\
\hline
\end{tabular}

* Slopes are derived from variable population data $(N=11-27) ; *$ Slopes are derived from four sample functions. 
pulse trains. While variability within subjects within a single test session was low, we suspect that variability over days was somewhat higher. We did not measure that variability systematically in this study, but we know from previous work that variability from day to day for electrical stimulation can be several $\mathrm{dB}$ for some subjects. This day to day variability could influence comparisons of data from different experiments such as Experiments 1 and 2 . However, if this variability is random, resulting in increased thresholds for some subjects and decreased thresholds for others, we would expect it to have less influence on comparisons of mean thresholds for all subjects across the two experiments.

Psychophysical techniques can also influence the levels of measured thresholds. Since we used different procedures for Experiments 1 and 2, caution must be used in comparing data from these two experiments. Comparisons of the two methods, made in Experiment 4 , suggest that these effects should be small. However, those comparisons involved a small number of subjects.

\section{Relation to Published Data}

Comparisons of these data with previously published thresholds (Table V) reveal a similarity with psychophysical thresholds from human and nonhuman primates, but important differences from thresholds for auditory nerve fibers from experiments in cats and squirrel monkeys. Threshold vs phase duration functions for detection of single electrical pulses by human subjects have not previously been reported, to our knowledge. However, thresholds for these stimuli have been published for nonhuman primates (Pfingst et al., 1991). Most of the data published previously for the monkey are for longer pulse durations than those tested here, but where the two data sets overlap, the slopes of the threshold vs phase duration functions are similar (Table V). Both data sets show a change in slope of the threshold vs phase duration functions at around 0.5 $\mathrm{ms}$ /phase. Note that in the nonhuman primate studies, slopes decreased as phase duration exceeded 5 ms/phase (Pfingst et al., 1991, Fig. 1 and Table II). However, we were unable to test stimuli of those long pulse durations with the Nucleus 22 Prosthesis. The absolute levels of single pulse thresholds for the human subjects reported here overlap those reported for the monkey but are higher on average. At about 1 $\mathrm{ms} /$ phase, thresholds for the human subjects in this experiment were between -5 and $-30 \mathrm{~dB}$ re $1 \mathrm{~mA}$ peak while those for the nonhuman primates reported by Pfingst et al. were between -20 and $-45 \mathrm{~dB}$. These differences in threshold levels could be due to a number of factors, including differences in electrode

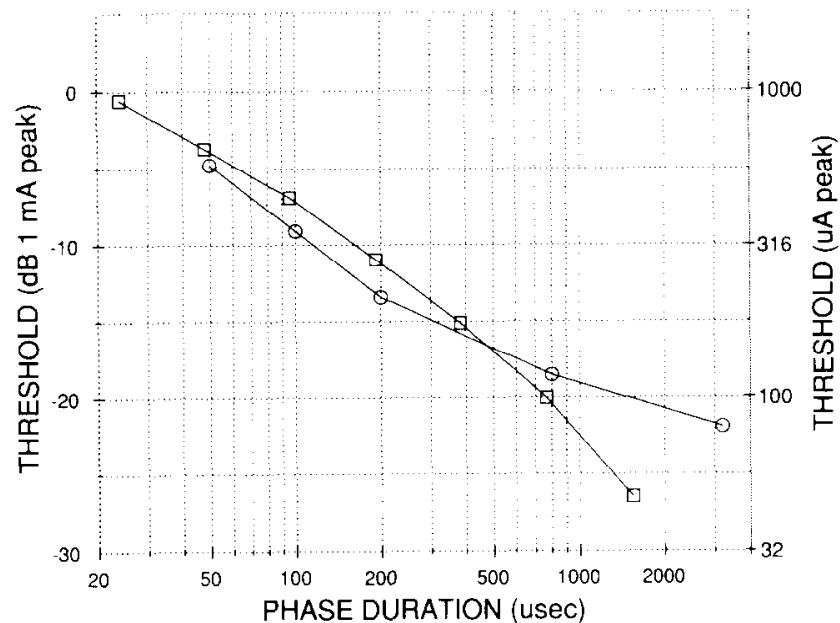

Fig. 8. Threshold vs phase duration functions for psychophysical and single fiber auditory nerve data. Square symbols denote mean psychophysical thresholds from this study. Mean single auditory nerve fiber thresholds for bipolar stimulation from Parkins and Colombo (1987) are represented by circles.

configurations and nerve survival patterns in the implanted ears.

Thresholds for pulse trains have been reported for human subjects by several investigators. Shannon et al. (1990) give thresholds for 2 subjects with Nucleus 22 implants, for pulse trains at $100 \mathrm{pps}$. Slopes for pulse durations of less than $0.5 \mathrm{~ms} /$ phase were similar to those found in the experiment reported in this paper (Table V). Shannon et al. measured thresholds only up to pulse widths of $909 \mu \mathrm{s} /$ phase, but there is evidence for most of the electrode pairs tested of a change in slope of the threshold vs phase duration functions near $0.5 \mathrm{~ms} /$ phase. In addition, Shannon (1983) measured thresholds for one subject with a UCSF implant for phase durations up to $8 \mathrm{~ms} /$ phase and this subject also showed a greater negative slope for pulses greater than $0.5 \mathrm{~ms} /$ phase.

Absolute values of the thresholds measured by Shannon et al. (1990) for the two subjects with Nucleus 22 prostheses are similar to those of subjects reported here, given corrections for differences in electrode separation. Thresholds for subjects tested with the UCSF electrode (Shannon, 1983) are lower than those for the subjects in this study but are within the range of values obtained from nonhuman primates.

Psychophysical thresholds are compared to single auditory nerve fiber thresholds in Table $\mathrm{V}$ and Fig. 8. Levels and slopes for single fiber threshold functions are similar to those of human threshold functions for phase durations less than $0.5 \mathrm{~ms} /$ phase. However, slopes of the neural functions are less steep for longer phase durations, while those for psychophysical detection are steeper. For phase durations longer than 0.5 
$\mathrm{ms}$, single neuron absolute threshold levels are higher on average than the psychophysical thresholds.

\section{Potential Mechanisms}

We assume that neurons must discharge in response to a stimulus if that stimulus is to be detected by the subject. The fact that subjects in this study described the signals they were detecting as auditory suggests that neural discharges underlying those perceptions were in the auditory pathway. Alternatively, this observation might be accounted for by some type of rcorganization of the brain.

Evidently, the neurons responsible for detection of signals with amplitudes of less than about $25 \mu \mathrm{A}$ peak have not been sampled in single fiber studies in animals, although psychophysical detection is possible at much lower currents (see Pfingst, 1988). Thus we hypothesize that there must be some, as yet unsampled, neurons that respond at lower levels of electrical stimulation. There are at least two alternative hypotheses regarding the characteristics of these unsampled neurons. (a) These neurons may have the same characteristics as the sampled neurons: i.e. they may act as leaky integrators of charge for which threshold current decreases as a function of phase duration at about 3 $\mathrm{dB} /$ doubling up to phase durations where the slope decreases due to accommodation. (b) They may have characteristics more similar to those of the psychophysical threshold-vs-phase duration functions. These hypotheses must be tested by single-unit recording. However, it seems unlikely that the threshold vs phase duration functions for individual fibers would have slopes of greater than $6 \mathrm{~dB} /$ doubling since that would imply that some mechanism other than integration of charge over time was responsible for those slopes. Perfect integration of charge over time would give a slope of $6 \mathrm{~dB} /$ doubling.

Hypothesis ' $a$ ' requires an additional mechanism to account for the differences in slope between the psychophysical and neural functions. If some neurons are responsive to long-phase-duration pulses at levels less than $25 \mu \mathrm{A}$, and if the thresholds of those neurons increase with decreasing phase duration at a rate of about $3 \mathrm{~dB}$ per halving of phase duration, then those neurons would discharge in response to short-phaseduration pulses at levels well below the psychophysical detection thresholds. This means that single spikes from those neurons would not be sufficient for psychophysical detection of short duration pulses. Rather, integration of multiple spikes, produced by the fibers at neural-suprathreshold levels, would be required for detection. Those multiple spikes could occur in a single fiber or across a population of fibers.

There are at least two known characteristics of the responses of auditory nerve fibers to electrical stimulation that could account for differences in the integra- tion of multiple spikes produced by short vs long single pulses, giving rise to threshold vs phase duration functions steeper than $6 \mathrm{~dB} /$ doubling. First, multiple spikes are more likely to occur within a single fiber if the pulse duration is long than if it is short (van den Honert and Stypulkowski, 1987; Parkins, 1989). Second, integration of spikes across fibers might be more favorable for long duration pulses, because all spikes are not evoked simultaneously with these pulses, as they are with shorter pulses (van den Honert and Stypulkowski, 1987). The assumption here is that if multiple neurons converge on a single output neuron and all of the inputs arrive simultaneously, the output will be only a single spike. However, if inputs are appropriately temporally dispersed, a train of output spikes might be generated.

At very long phase durations, neurons can not effectively integrate charge over the entire duration of the pulse, so the slope of the threshold vs phase duration function decreases. This phenomenon has been termed accommodation. If psychophysical detection is based on integration of spikes from multiple neurons we would anticipate some decrease in slope of the psychophysical detection threshold vs phase duration function at long phase durations, which has in fact been observed in previous studies that used longer phase durations than were tested here (Pfingst et al., 1991). However, the relationship of single fiber data to psychophysical data is complicated by the fact that both phases of the biphasic pulse could elicit spikes in a population of neurons. Thus as pulse duration increases, the temporal separation between spikes elicited by the two phases may increase, which could facilitate detection for reasons discussed above.

Several of the mechanisms proposed above assume that thresholds can be lowered by integration of multiple spikes occurring over time. It is well known that temporal integration can lower psychophysical detection thresholds for electrical stimulation (Shannon, 1989, Pfingst et al., 1991; this study, Experiment 3). This phenomenon occurs for stimulus durations up to at least $300 \mathrm{~ms}$ and thus is unlikely to be solely a property of the auditory nerve membrane. That is to say, some temporal integration must occur at sites central to the auditory nerve. Given this consideration, it is not unreasonable to suggest that integration of multiple discharges over time from either single fibers or across multiple fibers is in part responsible for the detection of single biphasic pulses as described above, and that the greater than $6 \mathrm{~dB} /$ doubling slopes of threshold vs pulse duration functions observed in psychophysical studies would be seen, not at the auditory nerve but only at higher centers in the auditory pathway. If this integration takes advantage of multiple spikes elicited by single pulses as well as spikes elicited by multiple pulse stimuli, this may explain the greater 
slopes of threshold vs phase duration functions for pulse trains with long phase duration pulses as compared to those with short phase duration pulses.

If the mechanisms described above include integration of spikes across fibers, then it is reasonable to expect that they will be sensitive to nerve survival patterns. The negative correlation between detection thresholds and speech perception performance observed in this study is consistent with the hypothesis that both measures are related to nerve survival. Factors affecting threshold, such as phase duration, can affect the strength of correlation between threshold and nerve survival (Pfingst and Sutton, 1984). In this study, correlations were stronger for longer phase duration pulses. Certainly many factors unlikely to be related to detection thresholds, such as cognitive abilities (e.g. Knutson et al., 1991), also contribute to speech perception. Length of auditory deprivation, has been correlated to speech perception scores (Kileny et al., 1991; Blamey et al., 1992). When scores of subjects with durations of profound deafness longer than 30 years were omitted, correlation coefficients of CID score vs threshold generally increased (Table IV). The highest correlation was observed using long phase duration $(1536 \mu \mathrm{s})$ pulse train thresholds with subjects $\mathrm{GE}$ and MO omitted from the sample. Such negative correlations were seen previously by Kileny et al. (1991) and by Eddington (personal communication). Curiously, however, Kuk et al. (1990) found positive correlation between threshold level and speech perception performance for NU6 words. Thus, further study is needed to understand the relationship between these variables. The relationship between threshold level and speech perception is not sufficiently strong that the threshold can be used by itself as a predictor of implant performance, though it may suggest a tendency toward better or poorer performance.

\section{Acknowledgements}

This work was supported by NIH-NIDCD grant DC00274. We express appreciation to Leslie Collins, Rebecca Coste, Chris Ellinger and Jim Heller for technical assistance and to Gregory Wakefield and Paul Kileny for providing test facilities. We particularly appreciate the time and cooperation of our subjects and their families. Finally, we acknowledge our debt to the work of the late John L. Kemink who was the physician to most of the subjects in this study. We miss him greatly.

\section{References}

Blamey, P.J., Pyman, B.C., Gordon, M., Clark, G.M., Brown, A.M., Dowell, R.C. and Hollow, R.D. (1992) Factors predicting postop- erative sentence scores in postlinguistically deaf adult cochlear implant patients. Ann. Otol. Rhinol. Laryngol. 101, 342-348.

Carhart, R. and Jerger, J. (1959) Preferred method for clinical determination and pure-tone thresholds. J. Speech. Hear. Disord. $24,220-345$.

Clark, G.M. (1987) The University of Melbourne-Nucleus multichannel cochlear implant. Adv. Otorhinolaryngol. 38, 1-189.

Frankenhaeuser, B. and Huxley, A.F. (1964) The action potential in the myelinated nerve fibre of Xenopus laecis as computed on the basis of voltage clamp data. J. Physiol. (London) 171, 302-315.

Gantz, B.J., Tyler, R.S., Knutson, J.F., Woodworth, G., Abbas, P., McCabe, B.F., Hinrichs, J., Tye-Murray, N., Lansing, C., Kuk, F. and Brown, C. (1988) Evaluation of five different cochlear implant designs: audiologic assessment and predictors of performance. Laryngoscope 98, 1100-1106.

Hill, A.V. (1936) The strength-duration relation for electric excitation of medullated nerve. Proc. R. Soc. Lond. 119, 440-453.

Javel, E., Tong, Y.C., Shepherd, R.K. and Clark, G.M. (1987) Responses of cat auditory nerve fibers to biphasic electrical current pulses. Ann, Otol. Rhinol. Laryngol. 96, Supplement 128, 26-30.

Kiang, N.Y.S. and Moxon, E.C. (1972) Physiological considerations in artificial stimulation of the inner ear. Ann. Otol. Rhinol. Laryngol. 81, 714-730.

Kileny, P.R., Zimmerman-Phillips, S., Kemink, J.L. and Schmaltz, S.P. (1991) Effects of preoperative electrical stimulability and historical factors on performance with multichannel cochlcar implant. Ann. Otol. Rhinol. Laryngol. 100, 563-568.

Knutson, J.F., Hinrichs, J.V., Tyler, R.S., Gantz, B.J., Schartz, H.A. and Woodworth, G. (1991) Psychological predictors of audiological outcomes of multichannel cochlear implants: preliminary findings. Ann. Otol. Rhinol. I aryngol. 100, 817-822.

Kuk, F.K., Tyler, R.S., Gantz, B.J. and Bertschy, M. (1990) Intensity operating range measures as predictors of word-recognition ability in cochlear implant subjects. Scand. Audiol. 19, 139-145.

Parkins, C.W. (1989) Temporal response patterns of auditory nerve fibers to electrical stimulation in deafened squirrel monkeys. Hear. Res. 41, 137-168.

Parkins, C.W. and Colombo, J. (1987) Auditory-nerve single-neuron thresholds to electrical stimulation from scala tympani electrodes. Hear. Res. 31, 267-286.

Pfingst, B.E. (1988) Comparisons of psychophysical and neurophysiological studies of cochlear implants. Hear. Res. 34, 243-251.

Pfingst, B.E.. De Haan, D.R. and Holloway, L.A. (1991) Stimulus features affecting psychophysical detection thresholds for electrical stimulation of the cochlea. 1: Phase duration and stimulus duration. J. Acoust. Soc. Am. 90, 1857-1866.

Pfingst, B.E., Glass, I., Spelman, F.A. and Sutton, D. (1985) Psychophysical studies of cochlear implants in monkeys: Clinical implications. In: R.A. Schindler and M.M. Merzenich (Eds.), Cochlear Implants, Raven Press, New York, pp. 305-321.

Pfingst, B.E. and Morris, D.J. (1992) Effects of stimulus frequency on detection of pulsatile electrical stimulation of the auditory nerve. Abstr. Assoc. Res. Otolaryngol., p. 7.

Pfingst, B.E. and Sutton, D. (1984) Relation of psychophysical thresholds for electrical stimuli to auditory nerve survival: Summary of results from 18 scala tympani implants. Abstr. Assoc. Res. Otolaryngol., p. 10.

Shannon, R.V. (1983) Multichannel electrical stimulation of the auditory nerve in man. I. Basic psychophysics. Hear. Res. 11, $157-189$.

Shannon, R.V. (1985) Threshold and loudness functions for pulsatile stimulation of cochlear implants. Hear. Res. 18, 135-143.

Shannon, R.V. (1989) A model of temporal integration and forward masking for electrical stimulation of the auditory nerve. In: J.M. Miller and F.A. Spelman (Eds.), Cochlear Implants. Models of the Electrically Stimulated Ear, Springer-Verlag, New York, pp. 187-203. 
Shannon, R.V., Adams, D.D., Ferrel, R.L., Palumbo, R.L. and Grandgenett, M. (1990) A computer interface for psychophysical and speech research with the Nucleus cochlear implant. J. Acoust. Soc. Am. 87, 905-907.

Skinner, M.W., Holden, L.K., Holden, T.A., Dowell, R.C., Seligman. P.M., Brimacombe, J.A. and Beiter, A.L. (1991) Performance of postlinguistically deaf adults with the wearable speech processor (WSP III) and mini speech processor (MSP) of the nucleus multielectrode cochlear implant. Ear. Hear. 12, 3-22. van den Honert, C. and Stypulkowski, P.H. (1987) Temporal response patterns of single auditory nerve fibers elicited by periodic electrical stimuli. Hear. Res. 29, 207-222.

Wilson, B.S., Finley, C.C., Lawson, D.T., Wolford, R.D., Eddington. D.K. and Rabinowitz, W.M. (1991) Better speech recognition with cochlear implants. Nature 352, 236-238. 